\title{
Subgrid-Scale Dynamics of Water Vapour, Heat, and Momentum over a Lake
}

\author{
Nikki Vercauteren · Elie Bou-Zeid • Marc B. Parlange • \\ Ulrich Lemmin • Hendrik Huwald • John Selker • \\ Charles Meneveau
}

Received: 21 February 2008 / Accepted: 17 June 2008 / Published online: 5 July 2008

(C) Springer Science+Business Media B.V. 2008

\begin{abstract}
We examine the dynamics of turbulence subgrid (or sub-filter) scales over a lake surface and the implications for large-eddy simulations (LES) of the atmospheric boundary layer. The analysis is based on measurements obtained during the Lake-Atmosphere Turbulent EXchange (LATEX) field campaign (August-October, 2006) over Lake Geneva, Switzerland. Wind velocity, temperature and humidity profiles were measured at $20 \mathrm{~Hz}$ using a vertical array of four sonic anemometers and open-path gas analyzers. The results indicate that the observed subgrid-scale statistics are very similar to those observed over land surfaces, suggesting that the effect of the lake waves on surface-layer turbulence during LATEX is small. The measurements allowed, for the first time, the study of subgrid-scale turbulent transport of water vapour, which is found to be well correlated with the transport of heat, suggesting that the subgrid-scale modelling of the two scalars may be coupled to save computational resources during LES.
\end{abstract}

Keywords Large-eddy simulation · Non-linear model · Smagorinsky model · Turbulent Prandtl number · Turbulent Schmidt number

N. Vercauteren · E. Bou-Zeid · M. B. Parlange $\cdot$ U. Lemmin $\cdot$ H. Huwald

School of Architecture, Civil and Environmental Engineering, École Polytechnique Fédérale de Lausanne-EPFL, Lausanne, Switzerland

E. Bou-Zeid ( $ه)$

Department of Civil and Environmental Engineering, Princeton University, Princeton, NJ 08544, USA e-mail: ebouzeid@princeton.edu

J. Selker

Department of Biological and Ecological Engineering, Oregon State University, Corvallis, OR 97331, USA

C. Meneveau

Department of Mechanical Engineering and Center for Environmental and Applied Fluid Mechanics, The Johns Hopkins University, 3400 N Charles St., Baltimore, MD 21218, USA 


\section{Introduction}

Quantifying the interaction of the atmosphere with an underlying water surface is of importance for many scientific endeavours such as improving lake evaporation models, developing surface parameterizations for numerical simulations, studying the ecological implications of climate change on water bodies, and understanding the local-scale atmospheric dynamics in coastal areas (Brutsaert 1982; Parlange et al. 1995; Edson et al. 2007). However, wateratmosphere interaction has generally received less attention than land-atmosphere interaction (DeCosmo et al. 1996), mainly due to logistical difficulties in operating field studies over water surfaces. The Lake-Atmosphere Turbulent EXchange (LATEX) field measurement campaign was designed to help bridge this gap and address some of the issues listed above. The experiment took place from mid-August through late October 2006, $100 \mathrm{~m}$ from the shore, in a 3-m deep section of Lake Geneva, Switzerland (Fig. 1). The primary instrumentation consisted of a vertical array of four sonic anemometers and four open-path $\mathrm{H}_{2} \mathrm{O} / \mathrm{CO}_{2}$ analyzers.

The main goal of LATEX was to study the dynamics of small-scale turbulence over the lake. The increasing interest in small-scale turbulence over the past decade is directly linked to the emergence of large-eddy simulation (LES) as a leading technique for the simulation of high Reynolds number ( $R e$ ) turbulent flows in the atmosphere (Moeng 1984; Albertson and Parlange 1999a, b; Wood 2000; Bou-Zeid et al. 2004, 2007; Patton et al. 2005; Stoll and Porte-Agel 2006), oceans (Shen and Yue 2001; Sullivan et al. 2007), rivers (Bradbrook et al. 2000; Keylock et al. 2005), as well as in engineering systems (Piomelli 1999; Sagaut 2003). In LES, all the large scales that one can computationally afford to capture on a numerical grid (the resolved scales) are simulated. The dynamics of the small turbulent eddies cannot be captured and have to be parameterized using subgrid-scale (SGS) turbulence models. The results of LES are sensitive to the SGS model formulation and hence significant research efforts have focused on understanding the dynamics of small-scale turbulence and on developing and testing various SGS models for applicability under different conditions.

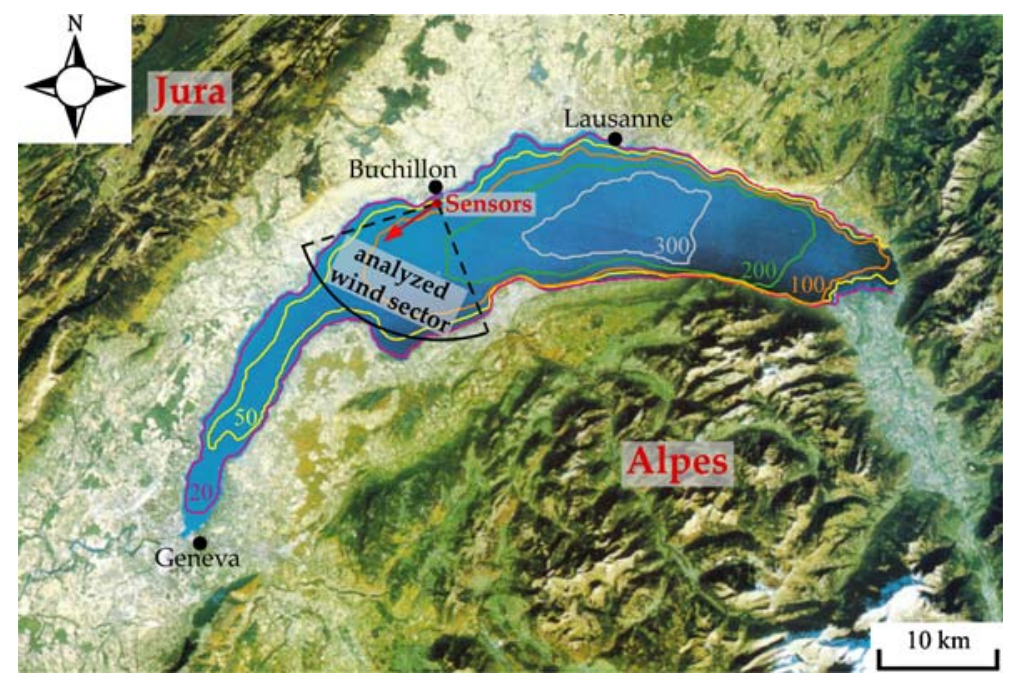

Fig. 1 Location and orientation of sensors, analyzed wind sector, and the bathymetry (m) of Lake Geneva, adapted from public domain satellite image (NASA World Wind) and bathymetry data (SwissTopo) 
Fig. 2 Significant wave height versus wavelength during the experiment as measured by a submersible level transducer (Pressure systems Inc. model 735; $0.05 \%$ accuracy) mounted $1.15 \mathrm{~m}$ below the water surface

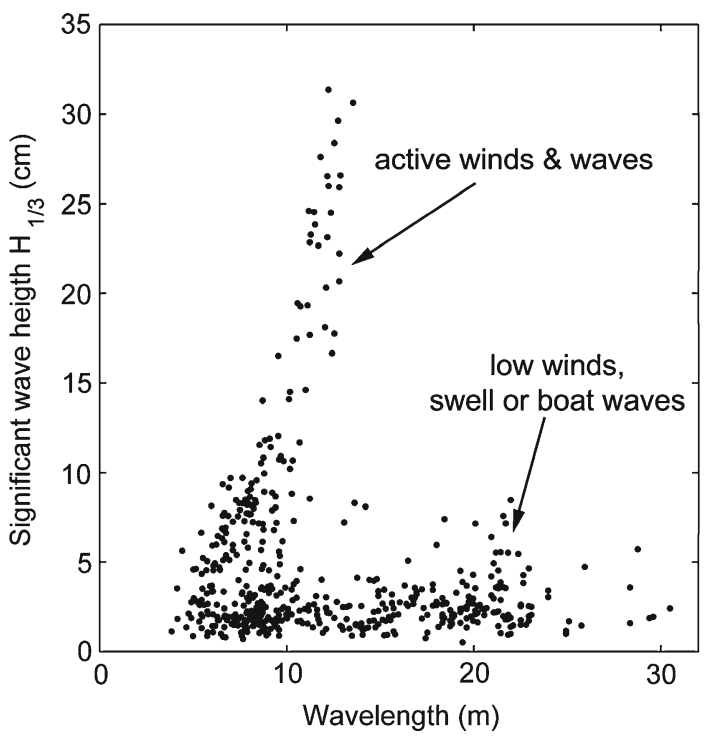

The focus of our paper is on the dynamics of these small turbulent scales over the water surface of the lake. Specifically, the aim is to answer the following two questions: (1) How are SGS dynamics over the lake different from SGS dynamics over relatively flat land surfaces? (2) How correlated are SGS fluxes and dissipations of water vapour (a passive scalar) and heat (an active scalar) (Katul and Parlange 1995; Assouline et al. 2008), and if well correlated, could their modelling be coupled/combined in LES.

To answer the first question, one has to look at the effect of water surface dynamics on atmospheric turbulence at the measurement height, noting that during LATEX the average upwind fetch was $15 \mathrm{~km}$ (Fig. 1). The lake is only $3 \mathrm{~m}$ deep at the measurement site and turbulence in the water body is relatively weak and mainly generated by the shear at the air-water interface. For wind speeds between $1 \mathrm{~m} \mathrm{~s}^{-1}$ and $10 \mathrm{~m} \mathrm{~s}^{-1}$, the heights of the waves had a median of about $0.03 \mathrm{~m}$ and rarely exceeded $0.2 \mathrm{~m}$ (note that atmospheric measurements start at a height of $1.65 \mathrm{~m}$ ); wavelengths were about $8 \mathrm{~m}$ on average. Figure 2 shows the significant wave height $H_{1 / 3}$, defined as the average of the highest third of the waves, versus wavelength. The wave speed was about $70 \%$ of the wind speed, and the wind speed and the friction velocity $u_{*}$ reached up to $8 \mathrm{~m} \mathrm{~s}^{-1}$ and $0.3 \mathrm{~m} \mathrm{~s}^{-1}$ respectively. During low winds (lower branch in Fig. 2), swell (and occasionally boats) generate much longer waves but their heights never exceed $0.05 \mathrm{~m}$. These features suggest that the dynamics of the water surface are relatively weak, mainly local, and atmosphere-driven. Therefore, the LATEX experimental site allows the study of a relatively simple case of atmosphere-water interaction where the effect of the waves on atmospheric turbulence is minimal.

This sets LATEX apart from the only other field experiment designed to study SGS dynamics over water surfaces, the Ocean Horizontal Array Turbulence Study (OHATS, Sullivan et al. 2006) which took place over the Atlantic Ocean, off the coast of Massachusetts. The OHATS set-up consisted of two vertically-separated horizontal arrays, each having nine sonic anemometers. Results from that study indicate that the interaction of the atmosphere with the underlying ocean waves in OHATS is an important factor to consider, mainly due to low wind speeds and the presence of swell generated far from the measurement site (Sullivan et al. 2006). Under such conditions, the ocean dynamics are not a response to, 
or in equilibrium with, the local atmospheric dynamics and the effect of the high ocean waves on atmospheric turbulence is important (DeCosmo et al. 1996; Sullivan et al. 2000). Proper understanding and modelling of air-water interactions obviously require a thorough investigation of the two limiting dynamics illustrated by LATEX and OHATS, as well as intermediate regimes. A discussion of different water surface dynamic regimes subjected to the disrupting effects of turbulence and the stabilizing effects of gravity and surface tension can be found in Brocchini and Peregrine (2001a,b).

A novel aspect of LATEX was the high-frequency measurements of water vapour concentration fluctuations in the air allowing computation of SGS parameters, testing of SGS models, and derivation of optimal model coefficients for water vapour. The second open question of the paper can then be addressed by comparing them with the results for heat. The findings are of particular importance since little has been reported in the literature on the SGS dynamics of water vapour, as opposed to numerous studies on heat and momentum dynamics.

A short review of SGS equations and models is presented in the following section, including the formulations of the two SGS models tested in this paper, the Smagorinsky model and the non-linear model. Section 3 describes the experimental site and set-up. The datasets and the processing needed to compute SGS parameters are detailed in Section 4. Section 5 presents transects through the flow (at the resolution afforded by the set-up) illustrating the turbulent structures and their relation to the filter size. The optimal coefficients for the two models and their dependence on filter size and stability are discussed in Section 6. In Section 7, we test the ability of the models to reproduce the measured fluxes and dissipations, and a comparative analysis of heat and water vapour SGS physics is included in Section 8. We conclude with a synthesis of the results addressing the two questions raised above.

\section{Subgrid-Scale Physics}

Large-eddy simulation of high Reynolds number flows is computationally possible because it resolves only the large eddies that contain most of the energy and perform most of the turbulent transport of momentum and scalars. The large eddies are explicitly captured by solving prognostic equations for their motion. These equations are obtained by applying a filtering operation to the full Navier-Stokes equations to remove the contribution of the unresolved eddies that are smaller than the grid size. The dynamics of these small eddies cannot be captured; however, their effect on the resolved eddies cannot be totally removed by the filtering operation due to non-linear scale interactions. This effect appears in the filtered Navier-Stokes equations as the divergence of the subgrid-scale (SGS) stress defined as:

$$
\sigma_{i j}=\widetilde{u_{i} u_{j}}-\widetilde{u}_{i} \widetilde{u}_{j},
$$

where $u_{i}, u_{j}$, and $u_{k}$ are the velocity components in three directions and the tilde $(\sim)$ denotes the filtering operation. In practice, the isotropic (hydrostatic) part of $\sigma_{i j}$ is lumped with the pressure term and the equations are written with the divergence of the anisotropic (deviatoric) part

$$
\tau_{i j}=\sigma_{i j}-\frac{1}{3} \sigma_{k k} \delta_{i j}
$$

Similar issues arise with the filtered scalar transport equations where the effect of the unresolved scales appears as the divergence of the subgrid-scale fluxes defined as:

$$
q_{i}=\widetilde{u_{i} b}-\widetilde{u_{i}} \widetilde{b}
$$

$b$ being the temperature or the concentration of a generic scalar such as water vapour. 
To close the system of filtered equations, a model for the subgrid-scale stress or flux is required. The results of large-eddy simulations are quite sensitive to this model especially in the vicinity of solid boundaries where the subgrid-scale fluxes are the most important and their physics are harder to parameterize due to the anisotropy of the flow (Meneveau and Katz 2000; Bou-Zeid et al. 2005). A large variety of subgrid-scale models have been proposed in the literature; however, the performance of a given model depends, for example, on the flow being simulated, grid resolution and performance assessment criteria. Usually, model validation is performed a posteriori, i.e. results from LES are compared to results for the same flow obtained through experimental measurements or through direct numeric simulation (DNS, which solves the full Navier-Stokes equations for all turbulence scales). Although this a posteriori assessment is the most pertinent test for LES and should be performed in model validation, it often does not reveal why a given SGS model works well or not for a given flow.

A complimentary approach for assessing SGS models is a priori testing. In this approach, highly-resolved turbulent fields from laboratory or field observations or DNS are filtered to split the turbulent fields into resolved and SGS fields (Meneveau 1994; Tong et al. 1999). The resolved fields are used to model SGS statistics as is done in LES (for example to compute the resolved strain rate tensor or the model coefficients as in Germano et al. 1991). These modelled statistics are then compared to the measured statistics obtained from the experimental or DNS SGS fields (Meneveau and Katz 2000; Porte-Agel et al. 2001b).

The most widely used model for the subgrid scales remains that of Smagorinsky (1963) or variants of it. The model relates the subgrid-scale stress to the resolved strain rate tensor $\tilde{S}_{i j}=0.5\left(\partial \tilde{u}_{i} / \partial x_{j}+\partial \tilde{u}_{j} / \partial x_{i}\right)$ via an eddy viscosity (hence the use of eddy model acronym later in this paper):

$$
\tau_{i j}^{e d d y}=-2 c_{S}^{2} \Delta^{2}|\widetilde{S}| \widetilde{S}_{i j}
$$

where $|\tilde{S}|=\left(2 \quad \tilde{S}_{i j} \quad \tilde{S}_{i j}\right)^{1 / 2}$ is the magnitude of the resolved strain rate tensor, $c_{s}$ is the Smagorinsky coefficient, and $\Delta$ is the filter scale. Similarly, the SGS flux of a scalar can be related to the gradient of that scalar via an eddy diffusivity,

$$
q_{i}^{e d d y}=-S c^{-1} c_{S}^{2} \Delta^{2}|\widetilde{S}| \frac{\partial \widetilde{b}}{\partial x_{i}},
$$

where $S c$ is the subgrid-scale turbulent Schmidt number for the scalar $b$ (or the Prandtl number, $P r$, for heat).

Another widely used set of SGS models is derived from the so-called similarity model (Bardina et al. 1980), an approach that postulates that the SGS stress is proportional to the turbulent stresses from the smallest resolved scales (between the grid scale $\Delta$ and a second test filter scale $\alpha \Delta$ ). A simpler implementation of the similarity model can be derived by performing a Taylor-series expansion of $\boldsymbol{u}$ yielding the non-linear model (Clark et al. 1979; Liu et al. 1994)

$$
\tau_{i j}^{n l}=C_{n l} \Delta^{2} \frac{\partial \tilde{u}_{i}}{\partial x_{k}} \frac{\partial \tilde{u}_{j}}{\partial x_{k}} .
$$

For the SGS flux of a scalar $b$, the non-linear model yields

$$
q_{i}^{n l}=C_{n l}^{b} \Delta^{2} \frac{\partial \tilde{u}_{i}}{\partial x_{k}} \frac{\partial \widetilde{b}}{\partial x_{k}} .
$$

The coefficients of the Smagorinsky and nonlinear models appearing in Eqs. 4-7 can be computed from LATEX data so that the mean measured SGS dissipation rates of turbulent 
kinetic energy $\left(\langle\Pi\rangle=-\left\langle\tau_{i j} \tilde{S}_{i j}\right\rangle\right)$ or scalar variances $\left(\langle\chi\rangle=-\left\langle q_{i} \partial \tilde{b} / \partial x_{i}\right\rangle\right)$ match the modelled ones (as done in Porte-Agel et al. (2000) and Kleissl et al. (2003) for example). We thus obtain the most suitable coefficients for the Smagorinsky model,

$$
\begin{aligned}
c_{s}^{2} & =\frac{-\left\langle\tau_{i j} \widetilde{S}_{i j}\right\rangle}{2(\Delta)^{2}\left\langle|\widetilde{S}| \widetilde{S}_{i j} \widetilde{S}_{i j}\right\rangle}, \\
\operatorname{Pr}^{-1} c_{s}^{2} & =\frac{-\left\langle q_{i}^{\text {heat }} \frac{\partial \widetilde{T}}{\partial x_{i}}\right\rangle}{\left\langle\Delta^{2}|\widetilde{S}| \frac{\partial \widetilde{T}}{\partial x_{i}} \frac{\partial \widetilde{T}}{\partial x_{i}}\right\rangle}, \\
S c^{-1} c_{s}^{2} & \left.=\frac{-\left\langle q_{i}^{H_{2} O} \frac{\partial{\widetilde{\rho_{v}}}_{x_{i}}}{\partial x_{i}}\right\rangle}{\left\langle\Delta^{2}|\widetilde{S}| \frac{\partial{\widetilde{\rho_{v}}}_{x_{i}}}{\partial x_{\rho_{v}}} \partial x_{i}\right.}\right\rangle
\end{aligned}
$$

and for the nonlinear model,

$$
\begin{gathered}
C_{n l}=\frac{\left\langle\tau_{i j} \widetilde{S}_{i j}\right\rangle}{\Delta^{2}\left\langle\frac{\partial \widetilde{u_{i}}}{\partial x_{k}} \frac{\partial \widetilde{u}_{j}}{\partial x_{k}} \widetilde{S}_{i j}\right\rangle} \\
C_{n l}^{\text {heat }}=\frac{\left\langle q_{i}^{\text {heat }} \frac{\partial \widetilde{T}}{\partial x_{i}}\right\rangle}{\left\langle\Delta^{2} \frac{\partial \widetilde{u_{i}}}{\partial x_{k}} \frac{\partial \widetilde{T}}{\partial x_{k}} \frac{\partial \widetilde{T}}{\partial x_{i}}\right\rangle} \\
C_{n l}^{H_{2} O}=\frac{\left\langle q_{i}^{H_{2}} O \frac{\partial \widetilde{\rho_{v}}}{\partial x_{i}}\right\rangle}{\left\langle\Delta^{2} \frac{\partial \widetilde{u_{i}}}{\partial x_{k}} \frac{\partial \widetilde{\rho_{v}}}{\partial x_{k}} \frac{\partial \widetilde{\rho_{v}}}{\partial x_{i}}\right\rangle}
\end{gathered}
$$

where $T$ is the temperature (K), $\rho_{v}$ is the water vapour concentration $\left(\mathrm{kg} \mathrm{m}^{-3}\right)$, and the angle brackets denote an averaging (in this paper time-averaging) operation. Even a coefficient that matches the dissipation rates on average cannot guarantee good results since it does not necessarily yield correct instantaneous energy dissipation or correct mean and instantaneous stresses and fluxes. Another important aspect is the alignment of the measured and modelled flux tensors (Clark et al. 1979; Meneveau and Katz 2000; Higgins et al. 2003, 2007) and the mean magnitude and the correlation of their individual components. The correlations of the measured and modelled fluxes and dissipations will be studied for both the Smagorinsky and the non-linear models; however, our focus here remains on understanding the SGS physics and answering the two questions introduced earlier, rather than testing the limits of a particular model.

\section{Site Description and Experimental Set-up}

The measurements over Lake Geneva in Switzerland were collected on a 10-m high tower, $100 \mathrm{~m}$ away from the northern shore of the lake. The measuring campaign lasted from mid August until late October 2006 (DOY 226 to DOY 298). Four sonic anemometers (Campbell Scientific CSAT3) and four open-path gas analyzers (LICOR LI-7500) measured wind speed, temperature, and humidity at 1.65, 2.30, 2.95 and $3.60 \mathrm{~m}$ above the water surface (Fig. 3). The four pairs of CSAT3/LI-7500 were arranged as a vertical array oriented towards the 

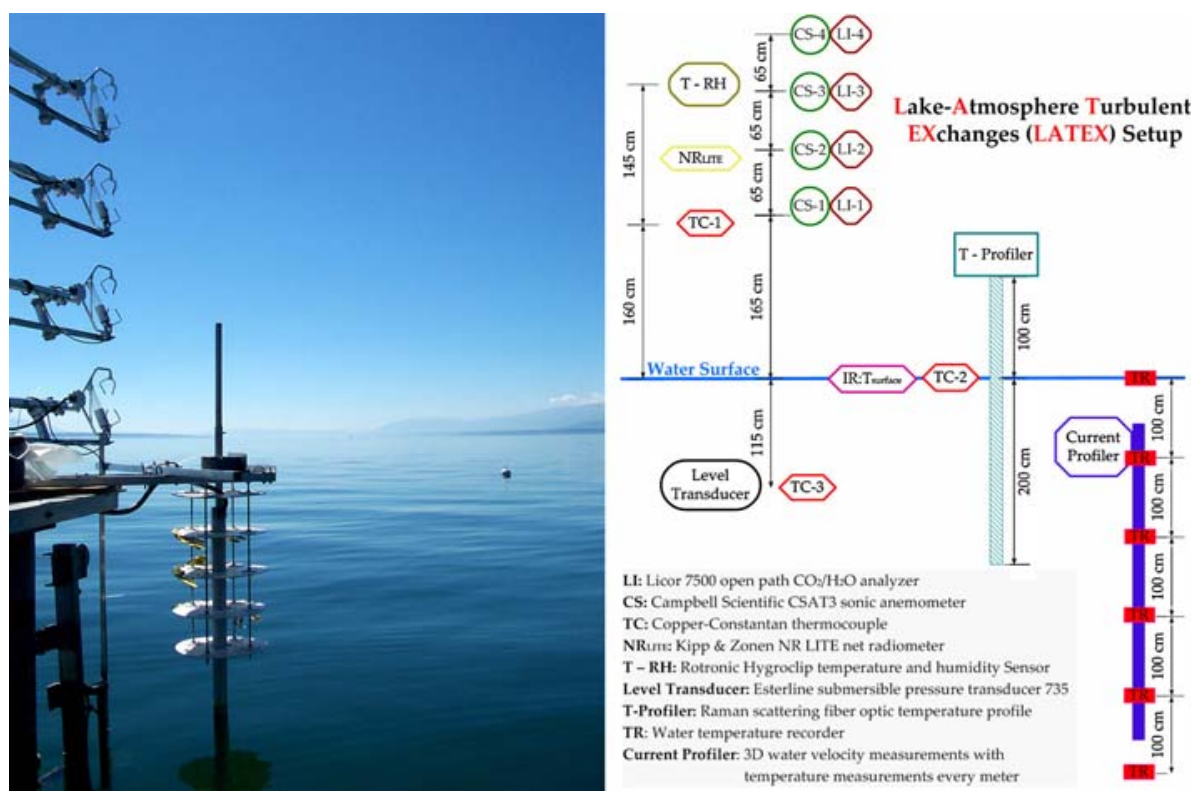

Fig. 3 Upstream view and experimental set-up of LATEX

south-west with a vertical separation of $0.65 \mathrm{~m}$. Only measurements with wind direction from the south and south-west were used to ensure that the minimum fetch remained $10 \mathrm{~km}$ and that the tower did not influence the measurements (see analyzed wind sector in Fig. 1). In this sector, the average upwind fetch is about $15 \mathrm{~km}$. Assuming that the internal equilibrium layer height is roughly equal to $1 / 100$ of the downstream distance (Brutsaert 1998; Bou-Zeid et al. 2004), the minimum fetch of $10 \mathrm{~km}$ ensures that the measurements are fully within this internal equilibrium layer of the lake.

Additional supporting measurements, depicted in Fig. 3, included (a) lake temperature obtained by a Raman-scattering fibre-optic temperature profiler with a 4-mm vertical resolution and about $0.01^{\circ} \mathrm{C}$ temperature resolution $(3 \mathrm{~m}$ range: $1 \mathrm{~m}$ above the water surface and $2 \mathrm{~m}$ below); (b) lake current from a profiler (Nortek); (c) net radiation (Kipp \& Zonen NR-Lite); (d) surface water temperature (thermocouple and Apogee Instruments IRTS-P infrared thermocouple sensor); (e) water temperature at $1.15 \mathrm{~m}$ depth (thermocouple); (f) air relative humidity and temperature (Rotronic hygroclip S3 at $3.05 \mathrm{~m}$ and a thermocouple at $1.60 \mathrm{~m}) ;(\mathrm{g})$ and wave height and speed (Pressure Systems Inc. submersible level transducer model $735 ; \pm 0.05 \%$ accuracy, mounted $1.15 \mathrm{~m}$ below the surface).

The raw data were collected at $20 \mathrm{~Hz}$ using a Campbell Scientific CR5000 data logger and all computations were done later, with pre-processing and data conditioning including triple rotation to correct the yaw, pitch, and roll misalignments of the sonic anemometers, linear detrending, and the density correction for fluxes (Webb et al. 1980). All instruments were purchased directly before the experiment and intercompared in the laboratory for calibration. In the laboratory, under zero wind conditions, the sonics had errors on the order of $0.021 \mathrm{~m} \mathrm{~s}^{-1}$ (maximum was $0.054 \mathrm{~m} \mathrm{~s}^{-1}$, manufacturer specification for offset is $0.04 \mathrm{~m} \mathrm{~s}^{-1}$ ). The standard deviation of the mean temperature readings of the four sonics in the laboratory was $0.53^{\circ} \mathrm{C}$ while LICOR readings were all within $3 \%$. For temperature and water vapour concentrations, we corrected the averages of the field measurements using the relative mean 
offsets of the different instruments measured in the laboratory. However, note that only deviations from the mean are used as we explain later in the paper, and hence these corrections will have an insignificant impact on the results.

\section{Computation of Gradients, Fluxes, and Dissipations}

We first note that the mean of the temperature and humidity over an averaging period (typically $15 \mathrm{~min}$ ) are subtracted from the signals before computing the subgrid fluxes and gradients of these scalars. This is needed because the accuracy of the means measured by the sonic anemometers (for temperature) and gas analyzers is not always satisfactory; these instruments are much better suited for the measurements of the turbulent deviations. This approach is in accordance with previous field studies of SGS physics (e.g. Porte-Agel et al. 2001b; Kleissl et al. 2003) and implies that the effects of mean gradients and interactions with the mean flow (for scalars) will not be captured; these effects should not be very critical at the turbulence scales we are studying. The components of the SGS stresses and fluxes are computed according to Eqs. 1 to 3. The filtering operation needed in these equations was performed in two dimensions (which was found to be equivalent to filtering in three dimensions with a filter size reduced by about $16 \%$ - Higgins et al. 2007). The $20 \mathrm{~Hz}$ data were first filtered in the vertical direction using a box filter of size $2 d z=1.3 \mathrm{~m}$; this operation was applied separately to the upper three sonic-Licor pairs and to the lower ones as depicted in Fig. 4 (left side). Taylor's frozen turbulence hypothesis was then employed to transform the two box-filtered time series into streamwise spatial series. This transformation allowed the application of a Gaussian filter (of any size $\Delta$ ) in the streamwise direction, yielding two spatial data series labelled as P1 (for the lower three sonic-Licor pairs) and P2 (for the upper three pairs) in Fig. 4 (right side). The gradients of filtered quantities were needed in all three directions, and the points around which these gradients were computed are points P1 in Fig. 4 coinciding with sonic-Licor pair number 2 at a height $z=2.3 \mathrm{~m}$. The vertical gradients were computed using first-order one-sided finite differences (FD) between points P1 and P2. The streamwise gradients were computed using a fourth-order centred differences (CD) scheme applied to the data stream P1, assuming Taylor's hypothesis. The $d x$ step was taken to be equal to $d z$ (see Kleissl et al. 2003). Tests performed for this study show that using second-order centred differences in the streamwise direction made no significant difference to the results.

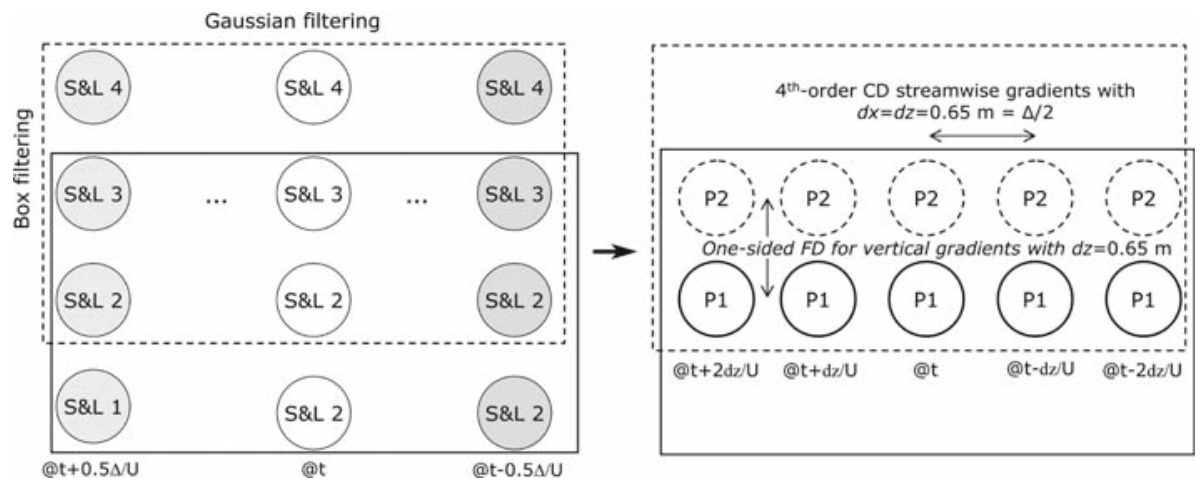

Fig. 4 Filtering and gradient computations in LATEX 
The cross-stream gradients could not be computed from the experimental settings since that would require at least two vertical arrays at two cross-stream locations. We can however approximate these terms by assuming turbulence isotropy or we can ignore them and effectively compute the two-dimensional (2D) surrogates (in the $x$ - $z$ plane) of the fluxes and gradients. The first approach (isotropy assumption) is usually preferable but it is problematic to apply for this dataset since, for momentum, it involves assuming turbulence isotropy in all three directions, which implies setting the missing contractions $\left\langle\tilde{S}_{12} \tilde{S}_{12}\right\rangle$ and $\left\langle\tilde{S}_{23} \tilde{S}_{23}\right\rangle$ equal to $\left\langle\tilde{S}_{13} \tilde{S}_{13}\right\rangle$ and $\left\langle\tau_{12} \tilde{S}_{12}\right\rangle$ and $\left\langle\tau_{23} \tilde{S}_{23}\right\rangle$ equal to $\left\langle\tau_{13} \tilde{S}_{13}\right\rangle$. These assumptions are not very realistic in a wall bounded flow at such a small distance from the surface since the computed vertical gradient $\tilde{S}_{13}$ and flux $\tau_{13}$ will be higher than the $(1,2)$ and $(2,3)$ components. Subtracting the means of $\tilde{S}_{13}$ and $\tau_{13}$ could be performed but will not solve the problem since the turbulent part will not be isotropic either. For scalars, isotropy can be assumed in the horizontal directions only but for the non-linear model this would transform some cross-product terms into streamwise quadratic terms (in the denominators of Eq. 9). To avoid these issues, the 2D surrogates of the fluxes and gradients are computed, i.e. only the $x$ and $z$ components are included in the analysis. This approach is justifiable on the basis that the vertical components are expected to be much larger close to the surface and the cross-stream terms contribution to the tensor contractions in Eqs. 8 and 9 will be small. The only crossstream term that we include is $d \tilde{v} / d y$ appearing in the strain rate tensor $\tilde{S}_{i j}$; this term can be accurately estimated from the continuity equation as: $d \tilde{v} / d y=-(d \tilde{u} / d x+d \tilde{w} / d z)$ and including it is expected to improve the estimate $\tilde{S}_{i j}$.

To check the effect of these assumptions, various tests were done (only feasible with the Smagorinsky model) and the effect of the treatment of the missing cross-stream gradients on the results was found to be minimal. When the isotropy assumption was tested, we noticed very small changes in the values of the optimal model coefficients for momentum and scalars, and a small decrease in the effect of stability on these coefficients compared to those obtained with the adopted 2D surrogate approach. This indicates either that the contribution of the missing terms is not important or that their trends are the same as the other terms that are computed and included (vertical and streamwise). The approach we selected is, however, the most realistic and gives the results that best match previous a priori studies. It gave indeed the same value of $c_{s}$ at neutral atmospheric stability as Kleissl et al. (2003, 2004), whereas assuming isotropy and removing the means of $\tilde{S}_{13}$ and $\tau_{13}$ gave values about $10 \%$ lower. In addition, it allows the comparison of the two SGS models.

The averaging operation (or the computation of correlations later in the paper) is always performed over 15-min time intervals. This was the base period for all runs and each run yields one data point (model coefficient, correlation, ....). For some aspects of the analysis, data from the 15-min runs were subsequently conditionally averaged based on atmospheric stability.

\section{Flow Structures}

To compare the filter scale to the size of the eddies at the measurement heights, we first look at vertical slices of the streamwise and vertical turbulent wind velocities, as well as the turbulent components of temperature and water vapour concentration depicted in Fig. 5. A low-pass filter of size $0.65 \mathrm{~m}$ is applied in the streamwise direction to match its frequency content with the vertical direction (following Porte-Agel et al. 2000); however, note that the actual resolution in the streamwise direction is typically much higher than the grid displayed in Fig. 5 (resolution = wind speed/sampling frequency). Well-defined flow structures can be 

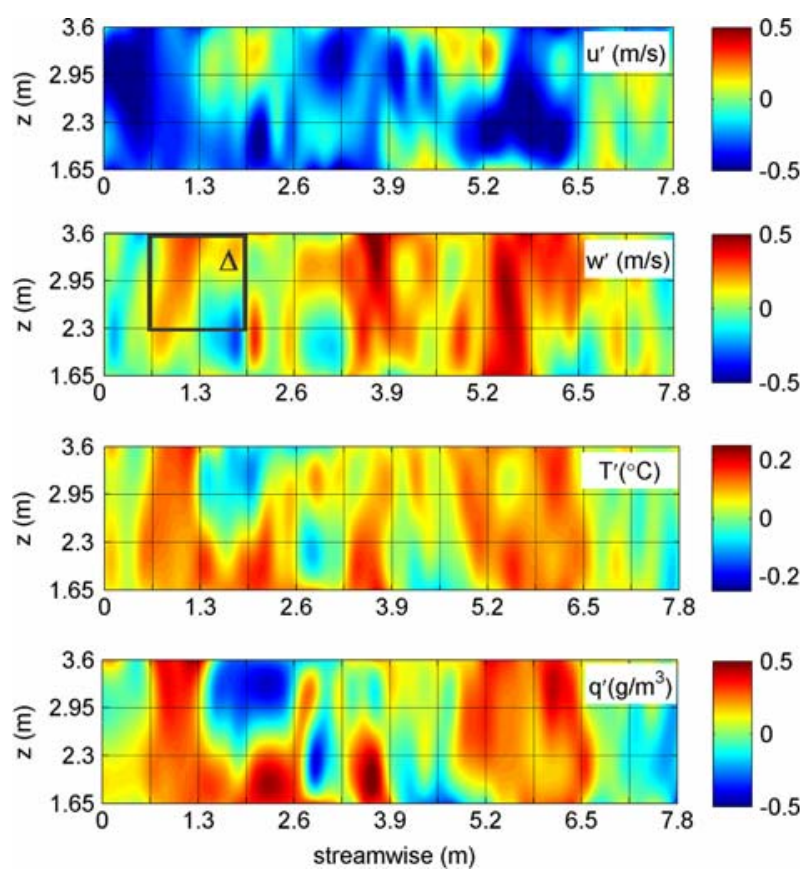

Fig. 5 Contour plots in a vertical plane of the turbulent components of the streamwise and vertical velocities, temperature, and water vapour concentration. The bold black square depicts the size of the filter. The horizontal grid lines show the vertical locations of the four sensors. The vertical grid lines show "virtual" streamwise locations of the sensors after applying Taylor's hypothesis and filtering out scales smaller than $d x=d z=0.65 \mathrm{~m}$ from the streamwise signal. The intersections of the grid lines can be interpreted as grid nodes where the flow is sampled to produce these slices. The turbulent components for this figure are computed based on 1-min averages to filter out the very large scales

identified. The strong correlation between $w^{\prime}, T^{\prime}$ and $q^{\prime}$ and their anti-correlation with $u^{\prime}$ are also visible indicating that these slices correspond to a period of upward heat flux $\left\langle w^{\prime} T^{\prime}\right\rangle$, water vapour flux $\left\langle w^{\prime} q^{\prime}\right\rangle$ and downward momentum flux $\left\langle w^{\prime} u^{\prime}\right\rangle$. Most of the measurements of LATEX correspond to periods of unstable stratification as measured by the stability parameter

$$
\frac{z}{L}=z \frac{\kappa g\left\langle w^{\prime} \theta_{v}^{\prime}\right\rangle}{-u_{*}^{3} \overline{\theta_{v}}},
$$

where $z$ is the measurement height, $L$ is the Obukhov length scale, $u_{*}$ is the friction velocity, $\theta_{v}$ is the virtual potential temperature, $\kappa$ is the von Karman constant, $g$ is the gravitational constant, and the brackets denote averaging, which is performed in time over $15 \mathrm{~min}$. Unstable stratification corresponds to negative values of $z / L$. We note that even when the sensible heat flux was downward suggesting stable stratification, the latent heat flux was upward and frequently balanced the stabilizing effect of the sensible heat flux. Values recorded for the sensible heat flux $H$ during the experiment ranged approximately between $-15 \mathrm{~W} \mathrm{~m}^{-2}$ and $40 \mathrm{~W} \mathrm{~m}^{-2}$, while the latent heat flux $L E$ was always positive and higher than the sensible heat flux but rarely exceeded $150 \mathrm{~W} \mathrm{~m}^{-2}$.

Figure 5 also illustrates that eddies with sizes on the order of $1 \mathrm{~m}$ are quite active in carrying vertical fluxes. This size can be compared to the basic filter size $\Delta=1.3 \mathrm{~m}$ used herein and depicted by the bold square in Fig. 5. The relative size of the structures is often comparable to 
or smaller than the filter size, which underscores the importance of SGS fluxes and dynamics in the vicinity of boundaries (in the friction layer) in LES of high Reynolds number flows where the viscous sublayer cannot be resolved (Pope 2004). In this limit, the SGS fluxes contribute significantly to the total fluxes; therefore, their function is not limited to dissipating turbulent kinetic energy (TKE) from the resolved scales. We found that the SGS contribution could be up to $40 \%$ of the total vertical fluxes of momentum and scalars.

\section{Dependence of SGS Model Coefficients on Filter Size, Height, and Stability}

The values for the model coefficients obtained from Eqs. 8 and 9 were computed and their variation with $z / L$ was investigated for various filter sizes $\Delta$. As previously discussed, most LATEX data were taken under unstable conditions and we hence restrict our analysis to variations of the coefficients with $z / L<0$. The averaging operations were performed (separately for the denominators and numerators of Eqs. 8 and 9) over $15 \mathrm{~min}$, resulting in 673 data points. Furthermore, we note that the box filter size in the vertical direction is limited to $1.3 \mathrm{~m}$ by the set-up of the experiment, whereas the horizontal Gaussian filter size can be varied; the filter size reported here is the effective size $\Delta=\left(\Delta_{\text {vertical }} \Delta_{\text {horizonal }}\right)^{1 / 2}$. The anisotropy of the filter resulting from this approach can affect the results, as will be discussed.

The Smagorinsky coefficient $c_{s}$ is expected to increase with increasing height to filter size ratio $z / \Delta$ to reflect the increasing mixing length $c_{s} \Delta$ (see, for example, Mason and Thomson 1992). On the other hand, it is often assumed that the effect of atmospheric stability on the Smagorinsky coefficient is negligible under unstable conditions (whereas the coefficient decreases sharply under stable conditions, see Kleissl et al. 2004). This assumption of a constant $c_{s}$ (for a given $z / \Delta$ ) under unstable conditions implies that increased mixing due to buoyancy in the unstable boundary layer has little effect on the turbulence structure. A review of previous studies (Porte-Agel et al. 2001a; Kleissl et al. 2004, for example) that did not report any sensitivity to increasing instability reveals that such studies were mostly restricted to the mildly unstable boundary layer $(-z / L<1)$. Other experimental studies (Chamecki et al. 2007) reported a continuous increase in $c_{s}$ as the instability (measured by using the Richardson number) increased.

During LATEX, higher values of $-z / L$ were observed due to the smoothness of the water surface (under low wind conditions) keeping $u_{*}$ low, while allowing the heat fluxes to reach values similar to those observed over land (latent and sensible heat fluxes sometimes exceeded $150 \mathrm{~W} \mathrm{~m}^{-2}$ and $40 \mathrm{~W} \mathrm{~m}^{-2}$, respectively). The resulting data indicate that $c_{s}$ values are significantly affected by stability. Figure 6 depicts the profile of $c_{s}$ as a function of $z / \Delta$ for different classes of stability: near neutral $(0<-z / L<0.1)$, unstable $(0.1<-z / L<1)$, and very unstable $(1<-z / L)$. We note that for $z / \Delta=1.77$, the filter is square; however, as we depart from this value, the filter becomes rectangular. The filter is also anisotropic due to the lack of explicit filtering in the cross-stream direction. To discriminate between the effects of filter anisotropy and filter size, we apply a correction for the anisotropy as proposed by Scotti et al. (1993). To apply this correction, we compute the effective cross-stream width from Higgins et al. (2007) who found that a $2 \mathrm{D}$ filter is equivalent to a $3 \mathrm{D}$ filter with a smaller effective size. Higgins et al. (2007) conclude that $0.86 \Delta_{2 \mathrm{D}}=\Delta_{3 \mathrm{D}}$ or $0.86(\Delta x \Delta z)^{1 / 2}=(\Delta x \Delta y \Delta z)^{1 / 3}$. This finding can then be used to compute the effective $\Delta y$ of a 3D filter that has the same $\Delta x, \Delta z$, and effective size of our 2D filter from $\Delta y=0.86^{3}(\Delta x \Delta z)^{1 / 2}=0.636(\Delta x \Delta z)^{1 / 2}$. Once we have the size of the filter in all three dimensions, we apply the correction factor as given by Eq. 16 in Scotti et al. (1993). The same results, but with $c_{s}$ values corrected for filter anisotropy, are presented in Fig. 7. The values of $c_{s}$ increase with the height-to-filter 
Fig. 6 Conditional averages of $c_{S}$, for three different classes of stability, versus $z / \Delta$

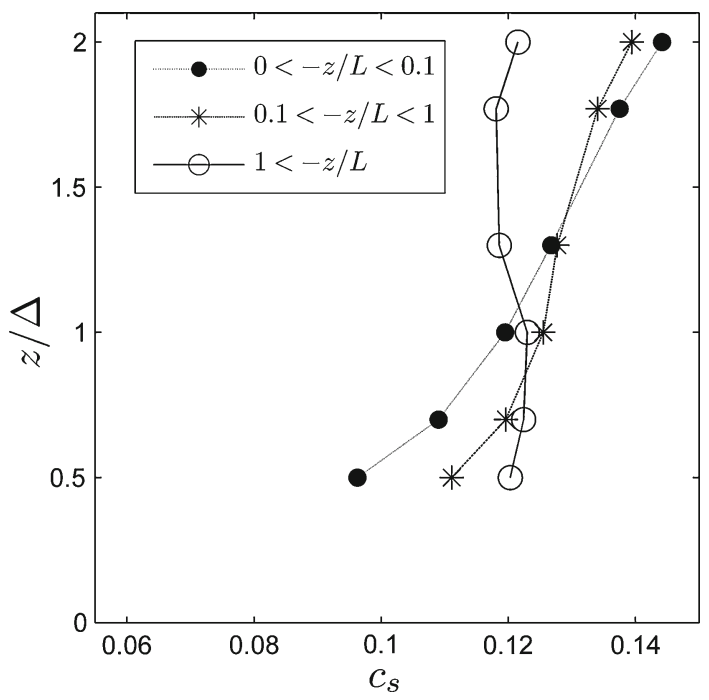

Fig. 7 The Smagorinsky parameter $c_{S}$ corrected for anisotropy (Scotti et al. 1993), versus $z / \Delta$ for three classes of stability

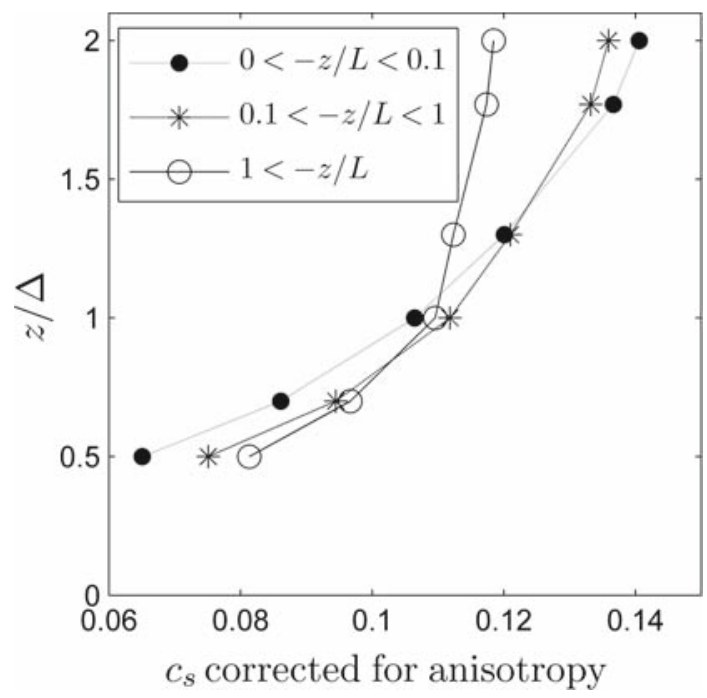

ratio $z / \Delta$ for all stabilities, as expected. The effect of increasing instability is to increase the integral scale and vertical mixing; this seems to flatten the profile of $c_{s}$ as the shear is reduced and the variations of SGS dissipation with filter scale decrease. Depending on the value of $z / \Delta, c_{s}$ might increase or decrease with stability.

The measured SGS Schmidt number is shown in Fig. 8 for $\Delta=1.3 \mathrm{~m}$ (square filter). The anisotropy correction for the scalar coefficients $\mathrm{Pr}^{-1} c_{s}^{2}$ and $S c^{-1} c_{s}^{2}$ can be shown to be equal to the correction for $c_{s}^{2}$; this is done using the isotropic turbulence estimate for the dissipation of scalar variance and arguments similar to Scotti et al. (1993). Hence, here we compute the scalar coefficients without correction and then compute $\mathrm{Pr}$ and $\mathrm{Sc}$ using the uncorrected values of $c_{s}^{2}$. This result implies that the SGS Prandtl and Schmidt numbers are not sensitive to filter anisotropy. 


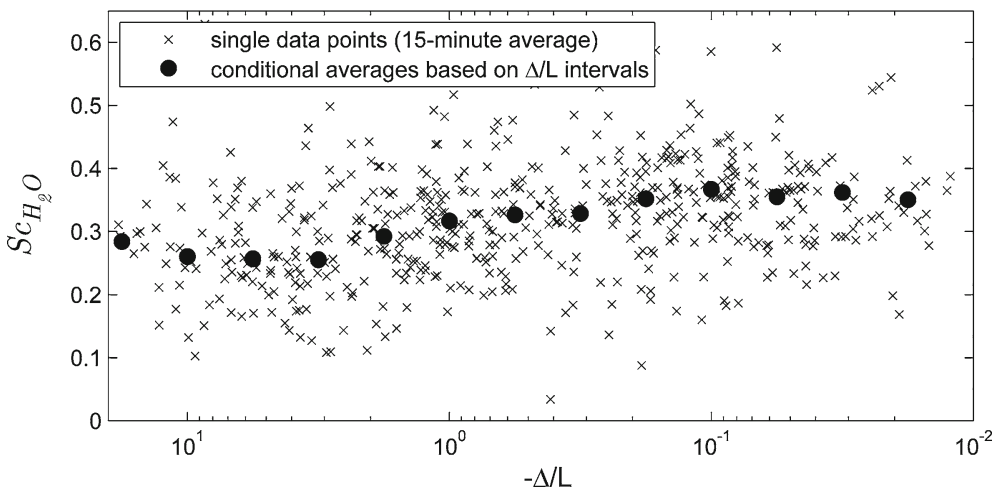

Fig. 8 The measured Schmidt number for water vapour as a function of stability

The approximate value of $S c$ is around 0.3 and shows a decrease from around 0.37 at near-neutral stabilities to around 0.27 under highly unstable conditions. Note that here we quantify stability in terms of $\Delta / L$ which is proportional to $z / L$ since we have a constant ratio $z / \Delta=1.77$. The value of 0.3 is very close to values reported in the few other studies that investigated the subgrid-scale Schmidt number through the dynamic SGS approach. Pitsch and Steiner (2000) found a value of $S c$ around 0.4 in (compressible) LES of a methane-air flame. Stoll and Porte-Agel (2006) obtained a value of about 0.3 away from the wall for a passive scalar in a neutral boundary layer, increasing as the wall was approached to around 0.4 at $z / \Delta=1$ (further increase was noticed at lower $z / \Delta$ but those points are likely to be significantly affected by the wall-model). A value of about 0.3 was again obtained through a dynamic model by Mitsuishi et al. (2003) for mass transfer across an air-water interface. While to our best knowledge, no previous a priori determination of the SGS Schmidt number for a passive scalar has been reported, the available evidence suggests that a value of the subgrid-scale $S c$ in the range of 0.3 to 0.4 holds well for a wide variety of flows. This is remarkable and underlines a basic strength of large-eddy simulation: the subgrid scales have universal properties and are easier to model than the full turbulence spectrum required in Reynolds-averaged Navier-Stokes (RANS) simulations. Note also that the value of the SGS Schmidt number of 0.3 is significantly lower than the 0.7 typically used in RANS to account for all turbulent scales, although the optimal value for RANS is known to be more flow dependent (see, for example, Yimer et al. 2002).

The measured Prandtl number depicted in Fig. 9 shows a trend similar to that of the Schmidt number with a more significant sensitivity to stability. $\operatorname{Pr}$ displays a clear decrease with increasing buoyancy, decreasing from around 0.38 at neutral stability to around 0.2 under highly unstable conditions. This indicates that the relative efficiency of heat transport increases in comparison with the efficiency of momentum transport as the surface layer becomes more unstable. The trend of increasing $\operatorname{Pr}$ as we approach neutral conditions is in agreement with the model used by Brown et al. (1994) and Mason and Brown (1999); however, the values do not match. That model predicts a value of $\operatorname{Pr} \approx 0.7$ at neutral stability decreasing to 0.44 under highly unstable conditions. The 0.38 value that we obtain at neutral stability is closer to the estimate of 0.47 by Mason (1989), who matched the subgrid-scale dissipation and its estimate based on the Kolmogorov theory in the inertial subrange (similar to the approach of Lilly 1967 , for $c_{s}$ ). We again note that the SGS Prandtl number is lower than the value of 0.7 typically used in RANS (Pope 2000) although, as we noted for the 


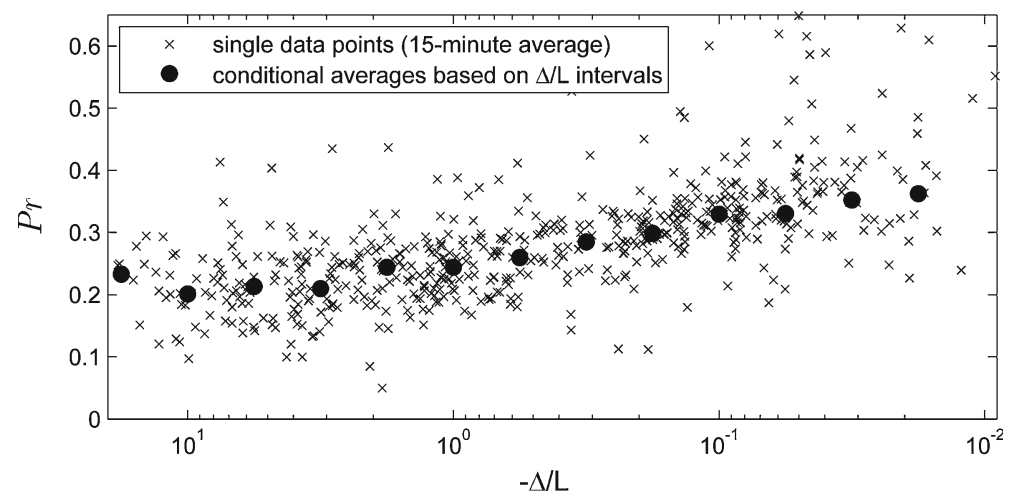

Fig. 9 The measured Prandtl number for heat as a function of stability

RANS Schmidt number, the RANS Prandtl number is highly flow dependent (see review in Kays 1994).

The variation of $\operatorname{Pr}$ with stability seems to be related to the active role of temperature since smaller variations are observed for the $S c$ of water vapour, a passive scalar. However, as the stability tends to neutral, the temperature field becomes almost homogeneous and the accuracy of the measurements of temperature variations tends to decrease. This explains the increased scatter of $\operatorname{Pr}$ values for single data points for small values of $-\Delta / L$ (Fig. 9) and indicates that more analysis is needed to confirm and explain the physical basis of the increase of $P r$ as the stability tends to neutral. This, however, will be left to future investigations based on results from LATEX and other field experiments.

The variations of Prandtl and Schmidt numbers with the height-to-filter size ratio $z / \Delta$ are shown in Figs. 10 and 11, for different stability ranges. A decrease of about $30 \%$ is observed for both numbers as the filter size decreases $(z / \Delta$ increases), and the effect of the filter size is hence considerably smaller than for the Smagorinsky coefficient, which doubled as $z / \Delta$ increased from 0.5 to 2 (Fig. 7). A very similar effect of $z / \Delta$ on $\operatorname{Pr}$ was observed by Porte-Agel et al. (2001b), where all three-dimensional components were available and the filter size varied by actually changing the experimental set-up, although their values were 5 to $10 \%$ higher than the values reported here. This decrease of $\mathrm{Pr}$ and $S c$ and the coinciding increase of $c_{S}$ as the filter size decreases indicates that the effect of the filter size on the SGS diffusivities of heat and water vapour $\left(-c_{s}^{2} \operatorname{Pr}^{-1} \Delta^{2}|\tilde{S}|\right.$ and $\left.-c_{s}^{2} S c^{-1} \Delta^{2}|\tilde{S}|\right)$ is smaller than its effect on the eddy viscosity for momentum $\left(-c_{s}^{2} \Delta^{2}|\tilde{S}|\right)$.

The variation of the non-linear model coefficients with filter size and stability was also investigated and we observed an increase of the coefficients with increasing $z / \Delta$. However, unlike with the Smagorinsky coefficients, there is no analytic correction for filter anisotropy, which makes it difficult to distinguish between the effect of the filter size and aspect ratio. Therefore we only report results obtained with the square filter, with a subsequent height-tofilter size ratio of $z / \Delta=1.77$. For this ratio, the non-linear model coefficients were found to be not very sensitive to variations in stability. The values of $C_{n l}$ for heat and water vapour were very close, both around 0.35 , a value close to that observed by Porte-Agel et al. (2001b, Fig. 11) for neutral conditions (they report an increase under stable conditions, which cannot be checked here). For momentum, the value of $C_{n l}$ was found to vary between 0.2 and 0.4. 
Fig. 10 Conditional averages of $\mathrm{Pr}$, for three different classes of stability, versus $z / \Delta$

Fig. 11 Conditional averages of Sc, for three different classes of stability, versus $z / \Delta$
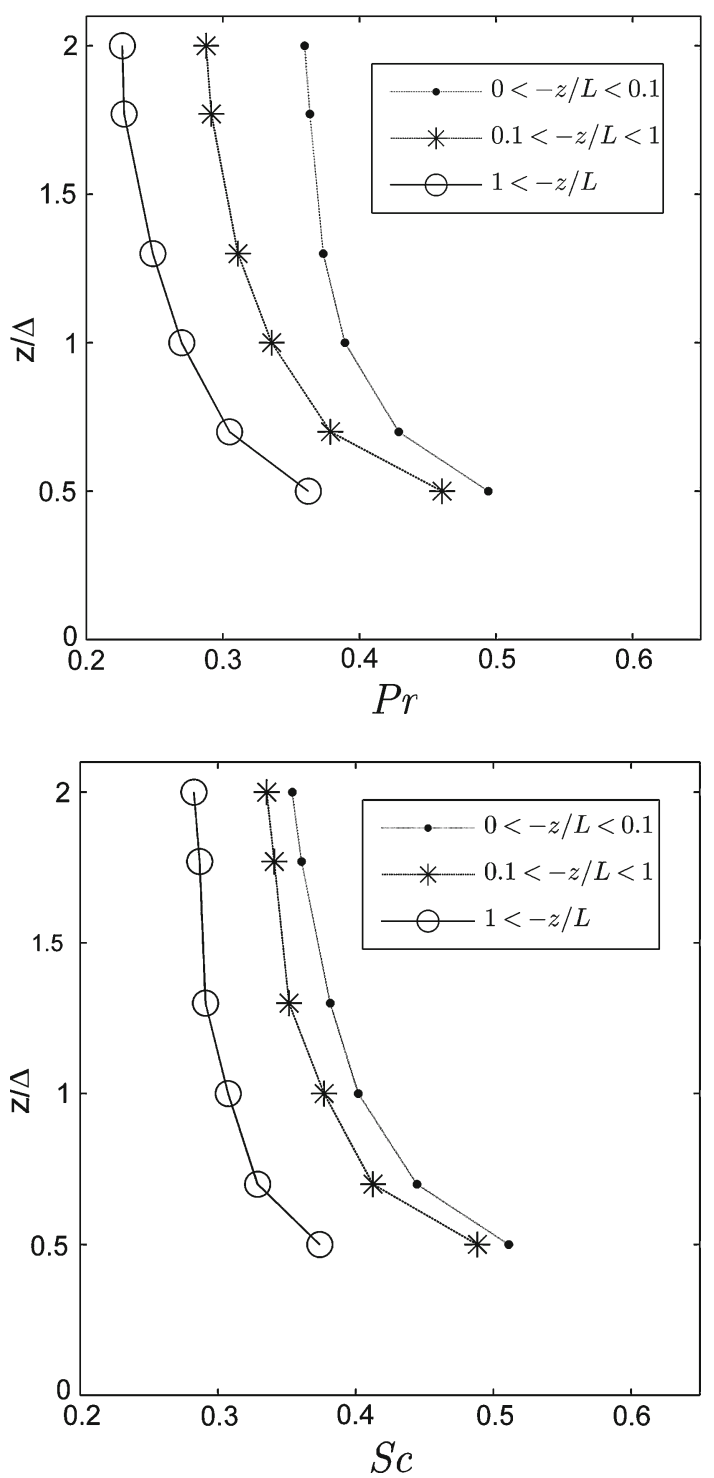

\section{Effect of Filter Size on the Modelled Fluxes and SGS Coefficients}

Even if the optimal coefficient is used in LES, the modelled fluxes will not be identical to the measured SGS fluxes. The models imply that the SGS stress tensor is aligned with the strain rate tensor (Smagorinsky model) or with the resolved stress tensor (similarity models) and that the model coefficients are constant over the averaging period or direction. In reality, the alignment assumption is not accurate (Liu et al. 1994; Tao et al. 2002; Higgins et al. 2003) and the optimal model coefficients vary considerably in space and time. The realistic aim of an SGS model is therefore to yield accurate SGS and resolved statistics, rather than to reproduce exact SGS fluxes locally in time and space. In spite of these arguments, an often used indicator of the realism of an SGS model is the correlation coefficient between individual components of the measured and modelled stress or flux tensors/vectors. As discussed in 
Fig. 12 Distribution of the correlations between measured and modelled vertical water vapour fluxes

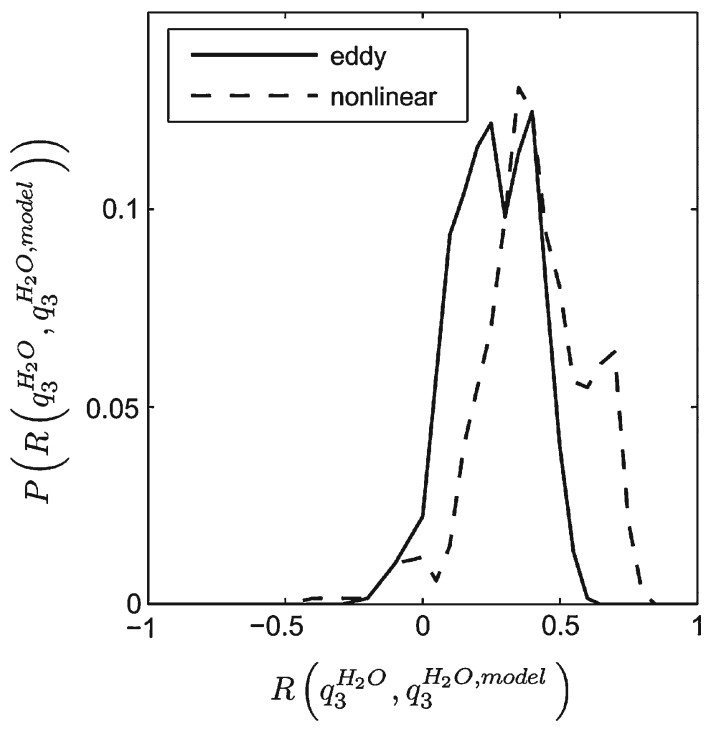

many references dealing with LES and SGS models (e.g. Meneveau and Katz 2000), good correlation coefficients are observed for SGS models that still do not perform well in LES (such as the similarity model alone). Therefore, results from such a priori correlation analysis must be interpreted carefully and we use them here to probe the alignment trends and physics of SGS fluxes rather than to validate the models. In addition, correlations have been reported in many other references dealing with SGS modelling and LES (e.g. O'Sullivan et al. 2001; Sullivan et al. 2003) and here we use the opportunity to compare the correlation coefficients trends for water vapour with previously observed trends for heat and momentum.

Another interesting parameter to analyze is the correlation of measured and modelled dissipation rates. Matching the dissipation averaged over $15 \mathrm{~min}$ allowed us to compute the optimal coefficients; however, the correlation coefficients of the instantaneous modelled and measured dissipation rates (within the 15-min run) indicates how well the model captures the unsteady dynamics of SGS dissipation. This correlation will hence reflect the variability of the model coefficient and is insensitive to the tensor misalignment.

All correlation coefficients are computed from the instantaneous values of fluxes or dissipation during a period of $15 \mathrm{~min}$ (yielding one correlation value per run). This computation is repeated for all runs yielding a dataset of correlation coefficients. The following results in this section present probability density functions (PDFs) of these correlation datasets or report their medians and standard deviations.

The probability distribution of the correlations between measured and modelled vertical fluxes of water vapour is depicted in Fig. 12 (for both models), for the square filter yielding $\mathrm{z} \Delta=1.77$. As frequently reported in the literature, the non-linear model performs better than the Smagorinsky model. The same results but for the correlations of variance dissipations are presented in Fig. 13. Again we observe that the non-linear model is better correlated with the measurements. Note that the correlations of the dissipations are significantly higher than the correlations of the fluxes.

Since the SGS dynamics depend strongly on the filter size $\Delta$ (Pope 2004), it is interesting to compare how the correlations vary with $\Delta$. However, the relative magnitude of $\Delta$ compared to the characteristic size of turbulent eddies is more important than the absolute value 
Fig. 13 Distribution of the correlations between measured and modelled dissipations of water vapour variance

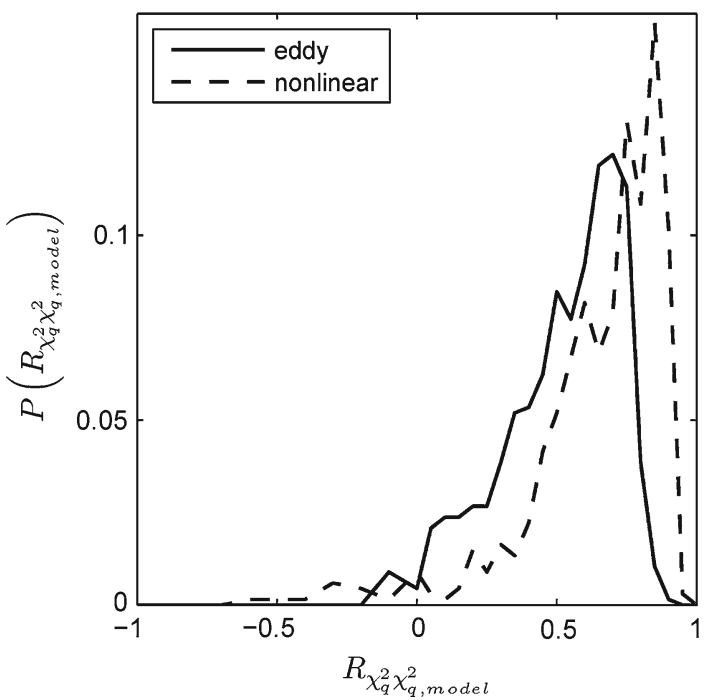

of the filter size. A surrogate of the integral scale of turbulence in wall-bounded flows is the height above the ground $z$; therefore, and we analyze the correlations as a function of $z / \Delta$. As previously noted, the stability parameter $z / L$ is also important and will affect the integral scale of turbulence; however, we do not assess the sensitivity of the correlations to stability here.

The medians and standard deviations of the distributions depicted in the figures above are listed for different $z / \Delta$ ratios in Table 1. Also included in the Table are the correlations for the streamwise fluxes of water vapour and for the vertical and streamwise fluxes of heat, with both models. The first part (four lines) of the Table reports the correlations for streamwise and vertical heat fluxes. All correlations seem to peak around $z / \Delta \approx 1$ and decrease for any changes in $z / \Delta$ thereafter. In the streamwise direction, the non-linear model performs much better than the eddy model; however, its advantage decreases at low $z / \Delta$. In the vertical direction, the performance of the two models is close and, surprisingly, the eddy model gives much higher correlation at low $z / \Delta$.

This comparative change in correlation behaviour as $z / \Delta$ decreases indicates that, as the grid size $\Delta$ increases and the LES filtering tends to the Reynolds average (close to walls), the validity of the eddy viscosity hypothesis for relating fluxes and strains improves (at least in comparison to the scale similarity hypothesis, which is more appropriate for inertial range turbulence far from walls). As discussed above, these near wall regions are typically the most critical for an SGS model (highest SGS fluxes and dissipation); the relatively higher correlations of the Smagorinsky model in these regions is a very significant result.

The second part of the Table (lines 5-8) presents the same results but for water vapour, and we observe the same peak in the correlations at about $z / \Delta \approx 1$ with correlations higher for the streamwise than the vertical flux component. The non-linear model again displays higher correlations in general except for low $z / \Delta$ values. Overall, the correlations for water vapour are slightly lower than for heat; this could be due to lower accuracy in the measurements of water vapour concentrations.

The last part of the Table reports the correlation between modelled and measured dissipation for heat and water vapour. As for fluxes, a peak of correlations is again clear at $z / \Delta \approx 1$, 
Table 1 Median (standard deviations in parentheses) of the correlations between the measured and modelled fluxes for different height to filter ratios

\begin{tabular}{lllllll}
\hline & $z / \Delta=0.5$ & $z / \Delta=0.7$ & $z / \Delta=1$ & $z / \Delta=1.3$ & $z / \Delta=1.77$ & $z / \Delta=2$ \\
\hline$q_{1}^{\text {heat }}, q_{1}^{\text {heat }, e d d y}$ & $0.40(0.18)$ & $0.42(0.17)$ & $0.39(0.15)$ & $0.35(0.13)$ & $0.28(0.11)$ & $0.26(0.10)$ \\
$q_{1}^{\text {heat }}, q_{1}^{\text {heat }, N L}$ & $0.54(0.31)$ & $0.65(0.26)$ & $0.74(0.22)$ & $0.75(0.22)$ & $0.69(0.22)$ & $0.66(0.21)$ \\
$q_{3}^{\text {heat }}, q_{3}^{\text {heat }, e d d y}$ & $0.43(0.14)$ & $0.47(0.13)$ & $0.49(0.13)$ & $0.48(0.14)$ & $0.44(0.14)$ & $0.42(0.13)$ \\
$q_{3}^{\text {heat }}, q_{3}^{\text {heat }, N L}$ & $0.24(0.17)$ & $0.39(0.16)$ & $0.58(0.17)$ & $0.68(0.18)$ & $0.61(0.18)$ & $0.54(0.17)$ \\
\hline$q_{1}^{H 2 O}, q_{1}^{H 2 O, e d d y}$ & $110.36(0.17)$ & $0.34(0.15)$ & $0.28(0.14)$ & $0.22(0.12)$ & $0.17(0.10)$ & $0.15(0.10)$ \\
$q_{1}^{H 2 O}, q_{1}^{H 2 O, N L}$ & $0.47(0.31)$ & $0.53(0.28)$ & $0.57(0.25)$ & $0.52(0.25)$ & $0.44(0.24)$ & $0.41(0.24)$ \\
$q_{3}^{H 2 O}, q_{3}^{H 2 O, e d d y}$ & $0.38(0.16)$ & $0.39(0.15)$ & $0.38(0.15)$ & $0.34(0.15)$ & $0.26(0.14)$ & $0.23(0.14)$ \\
$q_{3}^{H 2 O}, q_{3}^{H 2 O, N L}$ & $0.21(0.17)$ & $0.33(0.18)$ & $0.49(0.18)$ & $0.51(0.20)$ & $0.4(0.19)$ & $0.34(0.17)$ \\
\hline$\chi^{\text {heat }}, \chi^{\text {heat }, e d d y}$ & $0.58(0.21)$ & $0.64(0.18)$ & $0.72(0.19)$ & $0.75(0.20)$ & $0.70(0.19)$ & $0.66(0.19)$ \\
$\chi^{\text {heat }}, \chi^{\text {heat }, N L}$ & $0.64(0.32)$ & $0.75(0.26)$ & $0.86(0.21)$ & $0.90(0.20)$ & $0.84(0.22)$ & $0.80(0.20)$ \\
$\chi^{H 2 O}, \chi^{H 2 O, e d d y}$ & $0.53(0.23)$ & $0.59(0.21)$ & $0.64(0.21)$ & $0.65(0.21)$ & $0.57(0.21)$ & $0.52(0.20)$ \\
$\chi^{H 2 O}, \chi^{H 2 O, N L}$ & $0.59(0.32)$ & $0.70(0.29)$ & $0.80(0.25)$ & $0.82(0.23)$ & $0.73(0.23)$ & $0.66(0.23)$ \\
\hline
\end{tabular}

and temperature yields a higher correlation than water vapour. The overall correlations are higher than the correlation for fluxes; such increased correlations for a priori tests based on dissipation instead of fluxes was already observed in the first a priori tests in turbulence (Clark et al. 1979). The non-linear model correlates better with measurements but the difference with the eddy model decreases at low $z / \Delta$.

\section{Comparison Between Heat and Water Vapour SGS Dynamics}

Several studies of subgrid-scale heat fluxes can be found in the literature (Porte-Agel et al. 1998 for example), but little has been published about water vapour fluxes, as noted earlier. LATEX is the first experimental set-up that allows the computation of SGS fluxes and dissipation for water vapour and it is therefore of interest to compare the dynamics of water vapour fluxes and dissipation to heat fluxes and dissipation. The goal is to investigate under what conditions the dynamics are sufficiently correlated to realistically combine the modelling of the two SGS dynamics in LES. The gradients of the temperature and water vapour concentration would still be calculated separately of course but dynamic determination of the model coefficients (Germano et al. 1991) could be combined if the two components are well correlated.

Figure 14 depicts strong correlation between the vertical SGS heat and water vapour fluxes (similar correlation, not shown here, are obtained for streamwise fluxes). Figure 15 shows even higher correlations between the dissipation of heat and of water vapour variances (peaks above $85 \%$ ). Again, the effect of the filter size is investigated for sizes that do not entail significant filter anisotropy (recall that the square filter yields $z / \Delta=1.77$ ). One observes an increase in the correlation when the size of the filter increases. This indicates that at larger scales, where the dynamics are more averaged, the correlation between the two scalars is stronger. Conceptually, at the largest scale (the mean), the two fluxes are either upwards or 
Fig. 14 Distribution of the correlations (based on 15-min periods) of vertical heat and water vapour fluxes and variation with $z / \Delta$

Fig. 15 Distribution of the correlations (based on 15-min periods) of heat and water vapour variance dissipations and variation with $z / \Delta$
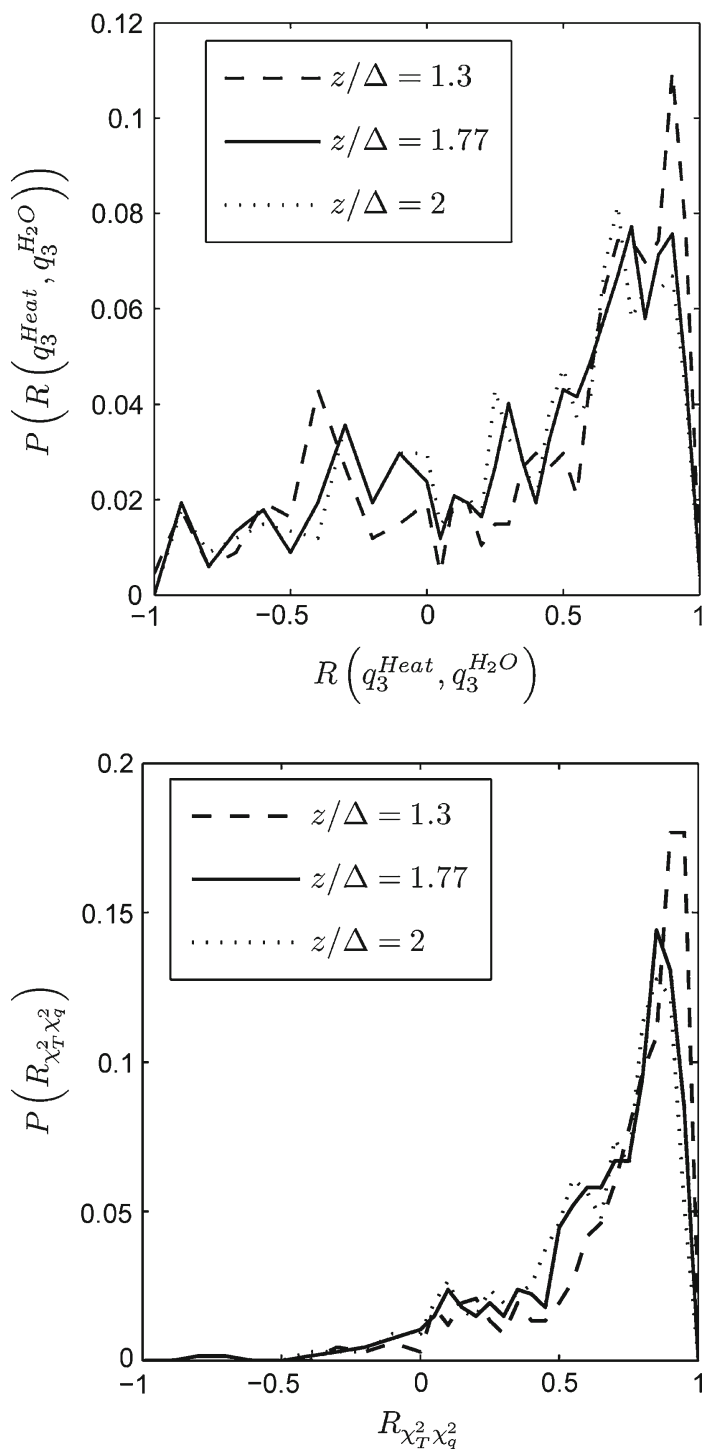

downwards and the correlation tends to 1 (or -1 if the mean fluxes are opposite). The large coherent structures maintain high correlations at large scales since they act in a similar way to convection. Further down in the turbulence cascade process, in the inertial subrange, the two scalars de-correlate. This trend was clearly visible in a coherence spectrum (which can be viewed as a frequency dependent correlation coefficient) of temperature and humidity (not shown here). The spectrum was above 0.9 for all the low frequencies and started to decrease only in the highest frequency decade (i.e. for scales smaller than $0.5 \mathrm{~s}$ ).

The strong correlations observed above encouraged us to look at the correlation between the model coefficients for water vapour and heat. As previously illustrated, these coefficients vary with stability and filter size and the question hence is whether their variations with these parameters are similar (only for unstable conditions with $z / L<0$ ). For the Smagorinsky 


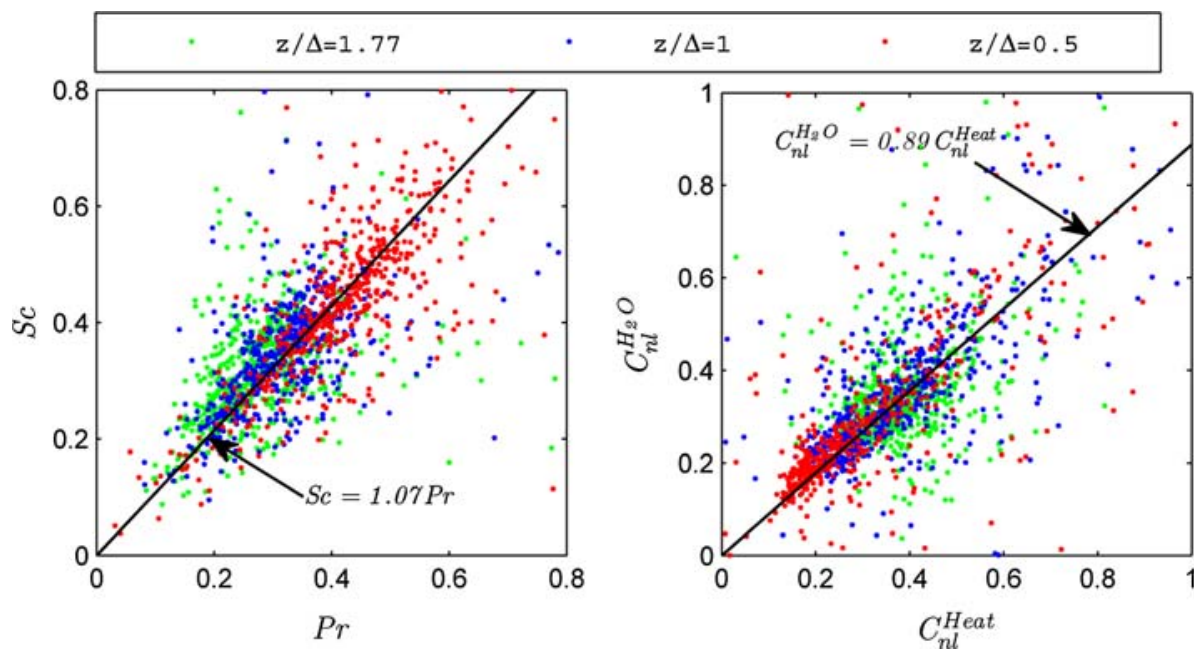

Fig. 16 Relations between model coefficients for heat and water vapour for three different filter sizes, on the left for the Smagorinsky model ( $S c$ versus $\mathrm{Pr}$ ) and on the right for the non-linear model

model, the plot of $\operatorname{Pr}$ versus $S c$ on the left-hand side of Fig. 16 shows a very robust linear relation between the two numbers that is not sensitive to stability or filter size. A bisquare linear fit for all the data yields a Schmidt number about $7 \%$ higher than the Prandtl number. The right-hand side of Fig. 16 depicts the relation of the two coefficients of the non-linear model and a bisquare linear fit (solid line in figure). We again observe good correlation between the two coefficients with $C_{n l}^{\text {heat }}$ roughly $11 \%$ higher than $C_{n l}^{H 2 O}$ according to the fit. For both models, one can note that the correlation improves considerably when the size of the filter increases; this is attributed to the increase in the correlation of humidity and temperature fields with scale, which we observed in a coherence spectrum as discussed above. We also observed (trend not illustrated in Fig. 16) that the correlation tends to improve as the atmospheric instability $(-z / L)$ increases. The total correlation coefficients for all filter sizes is about $65 \%$ for both models (ranging, for the coefficients of the non-linear model, from $33 \%$ for $z / \Delta=1.77$ to $73 \%$ for $z / \Delta=0.5$, and for the Smagorinsky model, from $38 \%$ at $z / \Delta=1.77$ to $75 \%$ at $z / \Delta=0.5$ ).

The good linear relations of the coefficients for heat and water vapour in both models suggest that a dynamic determination of only one of the coefficients could be sufficient (see Moin et al. (1991) and Porte-Agel (2004) for details on dynamic SGS models for scalars). The other coefficient can then be imposed based on the fits proposed here. This would save significant computational time and complexity in large-eddy simulation. Moreover, this suggests that dynamic coefficients for other passive tracers can also be computed from the coefficients of heat or water vapour via simple linear relations.

\section{Conclusion}

Measurements from the Lake-Atmosphere Turbulent Exchanges Experiment (LATEX) were analyzed to study specific aspects of subgrid-scale modelling for large-eddy simulation. The data analyzed here mainly consisted of high frequency measurements of wind, temperature and humidity that can be used to study actual SGS dynamics and compare them to modelled 
ones; we tested the Smagorinsky (eddy) model and the non-linear model. The main goals of the study were to: (1) compare SGS dynamics over the lake to the dynamics over flat land surfaces; (2) study SGS fluxes and dissipation of water vapour (a passive scalar) and compare them to fluxes and dissipation for heat (an active scalar). The following observations help us address the first goal of the paper:

1. The variations and values of the Smagorinsky model coefficient obtained over the lake (Fig. 7) were similar to those over land (Porte-Agel et al. 2001b; Kleissl et al. 2003). However, we detected a greater sensitivity of the model coefficients to stability mainly due to the fact that LATEX was able to reach much higher values of $-z / L$ than previous studies over land.

2. The SGS Prandtl and Schmidt numbers showed relatively mild variations with filter scale and stability ( $P r$ being more sensitive to stability than $S c$ ). These variations and the values of the two coefficients were similar to those obtained in other field experiments and through dynamic models for many types of flow.

3. The non-linear SGS model coefficients showed little sensitivity to stability and the values were comparable to values obtained in neutral stability over land (Porte-Agel et al. 2001a)

4. The correlations between measured and modelled SGS fluxes and dissipation (Table 1) were slightly lower than the values reported over land surfaces (Porte-Agel et al. 2001b); though this is likely due to the details of the analysis (mainly the vertical filtering and use of 2D surrogates in this paper).

From the above, we conclude that the turbulent subgrid scales over the lake obey the same dynamics as observed over land. No significant effect of the moving interface could be detected. Of course, the momentum and heat fluxes over the lake are different from those over land; however, variations in these parameters affect SGS dynamics in the same way over land and water. These findings can only be confirmed for water surfaces where the wave heights are low compared to the measurement height as is the case during LATEX (Fig. 2) and where the dynamics of the surface exchanges are mainly controlled by the atmospheric flow.

To address the question relating to the similarities between water vapour and heat SGS dynamics we note that:

1. The fluxes and dissipation of heat and water vapour show high correlations (Figs. 14 and 15)

2. The model coefficients for heat and water vapour are closely related for both the eddy and non-linear model and the relations are not sensitive to stability or filter size (Fig. 16).

3. The variations of the correlations, as a function of filter size, between measured and modelled fluxes and dissipation (Table 1) have the same trends for heat and water vapour, though the correlations are generally slightly higher for heat.

This suggests that the subgrid-scale dynamics for heat and water vapour are strongly correlated and dynamic SGS model implementations could compute the coefficient of one of the two scalars and impose the other coefficient based on the first one through the linear fits in Fig. 16 (dynamic coefficient determinations are rather expensive computationally). This result has been validated here only for unstable conditions and for surfaces where the temperature and water vapour concentration are well correlated at the large scales (sensible and latent heat exchanges at the lake surface are well correlated). It is not clear if the good correlation between heat and water vapour SGS dynamics will hold under stable conditions or over heterogeneous terrain with alternating hot-dry and cold-wet patches. 
Acknowledgements The authors would like the thank the Swiss National Science Foundation for its support for this work through grant number 200021-107910 and through the National Competence Center in Research on Mobile Information and Communication Systems (NCCR-MICS) under grant number 5005-67322. C. Meneveau is supported by the US National Science Foundation under grant EAR-0609690.

\section{References}

Albertson JD, Parlange MB (1999a) Surface length scales and shear stress: Implications for land-atmosphere interaction over complex terrain. Water Resour Res 35:2121-2132. doi:10.1029/1999WR900094

Albertson JD, Parlange MB (1999b) Natural integration of scalar fluxes from complex terrain. Adv Water Resour 23:239-252. doi:10.1016/S0309-1708(99)00011-1

Assouline S, Tyler SW, Tanny J, Cohen S, Bou-Zeid E, Parlange MB et al (2008) Evaporation from three water bodies of different sizes and climates: measurements and scaling analysis. Adv Water Resour 31:160-172. doi:10.1016/j.advwatres.2007.07.003

Bardina J, Ferziger JH, Reynolds WC (1980) Improved subgrid scale models for large eddy simulation. American Institute of Aeronautics and Astronautics Pap 80-1357

Bou-Zeid E, Meneveau C, Parlange MB (2004) Large-eddy simulation of neutral atmospheric boundary layer flow over heterogeneous surfaces: blending height and effective surface roughness. Water Resour Res 40:W02505. doi:10.1029/2003WR002475

Bou-Zeid E, Meneveau C, Parlange MB (2005) A scale-dependent Lagrangian dynamic model for large eddy simulation of complex turbulent flows. Phys Fluids 17:025105. doi:10.1063/1.1839152

Bou-Zeid E, Parlange MB, Meneveau C (2007) On the parameterization of surface roughness at regional scales. J Atmos Sci 64:216-227. doi:10.1175/JAS3826.1

Bradbrook KF, Lane SN, Richards KS, Biron PM, Roy AG (2000) Large eddy simulation of periodic flow characteristics at river channel confluences. J Hydraul Res 38:207-215

Brocchini M, Peregrine DH (2001a) The dynamics of strong turbulence at free surfaces. Part 1. Description. J Fluid Mech 449:225-254. doi:10.1017/S0022112001006012

Brocchini M, Peregrine DH (2001b) The dynamics of strong turbulence at free surfaces. Part 2. Free-surface boundary conditions. J Fluid Mech 449:255-290. doi:10.1017/S0022112001006024

Brown AR, Derbyshire SH, Mason PJ (1994) Large-eddy simulation of stable atmospheric boundary-layers with a revised stochastic subgrid model. Q J R Meteorol Soc 120:1485-1512. doi:10.1002/qj. 49712052004

Brutsaert W (1982) Evaporation into the atmosphere: theory, history, and applications. Reidel, Dordrecht, Holland, $299 \mathrm{pp}$

Brutsaert W (1998) Land-surface water vapor and sensible heat flux: spatial variability, homogeneity, and measurement scales. Water Resour Res 34:2433-2442. doi:10.1029/98WR01340

Chamecki M, Meneveau C, Parlange MB (2007) The local structure of atmospheric turbulence and its effect on the Smagorinsky model for large eddy simulation. J Atmos Sci 64:1941-1958. doi:10.1175/JAS3930.1

Clark RA, Ferziger JH, Reynolds WC (1979) Evaluation of sub-grid-scale models using an accurately simulated turbulent-flow. J Fluid Mech 91:1-16. doi:10.1017/S002211207900001X

DeCosmo J, Katsaros KB, Smith SD, Anderson RJ, Oost WA, Bumke K et al (1996) Air-sea exchange of water vapor and sensible heat: the humidity exchange over the sea (HEXOS) results. J Geophys Res-Oceans 101:12001-12016. doi:10.1029/95JC03796

Edson J, Crawford T, Crescenti J, Farrar T, Frew N, Gerbi G et al (2007) The coupled boundary layers and air-sea transfer experiment in low winds. Bull Amer Meteorol Soc 88:341-356. doi:10.1175/BAMS-88-3-341

Germano M, Piomelli U, Moin P, Cabot WH (1991) A dynamic subgrid-scale eddy viscosity model. Phys Fluids A 3:1760-1765. doi:10.1063/1.857955

Higgins CW, Parlange MB, Meneveau C (2003) Alignment trends of velocity gradients and subgrid-scale fluxes in the turbulent atmospheric boundary layer. Boundary-Layer Meteorol 109:59-83. doi:10.1023/ A: 1025484500899

Higgins CW, Meneveau C, Parlange MB (2007) The effect of filter dimension on the subgrid-scale stress, heat flux, and tensor alignments in the atmospheric surface layer. J Atmos Ocean Technol 24:360-375. doi:10.1175/JTECH1991.1

Katul GG, Parlange MB (1995) Analysis of land-surface heat fluxes using the orthonormal wavelet approach. Water Resour Res 31:2743-2749. doi:10.1029/95WR00003

Kays WM (1994) Turbulent Prandtl number - Where are we? J Heat Transfer 116:284-295. doi:10.1115/1. 2911398 
Keylock CJ, Hardy RJ, Parsons DR, Ferguson RI, Lane SN, Richards KS (2005) The theoretical foundations and potential for large-eddy simulation (LES) in fluvial geomorphic and sedimentological research. Earth Sci Rev 71:271-304. doi:10.1016/j.earscirev.2005.03.001

Kleissl J, Meneveau C, Parlange MB (2003) On the magnitude and variability of subgrid-scale eddydiffusion coefficients in the atmospheric surface layer. J Atmos Sci 60:2372-2388. doi:10.1175/15200469(2003)060<2372:OTMAVO >2.0.CO;2

Kleissl J, Parlange MB, Meneveau C (2004) Field experimental study of dynamic Smagorinsky models in the atmospheric surface layer. J Atmos Sci 61:2296-2307. doi:10.1175/1520-0469(2004)061<2296: FESODS $>2.0 . \mathrm{CO} ; 2$

Lilly DK (1967) The representation of small scale turbulence in numerical simulation experiments. In: IBM Scientific computing symposium on environmental sciences, White Plains, New York, pp 195-209

Liu SW, Meneveau C, Katz J (1994) On the properties of similarity subgrid-scale models as deduced from measurements in a turbulent jet. J Fluid Mech 275:83-119. doi:10.1017/S0022112094002296

Mason PJ (1989) Large-eddy simulation of the convective atmospheric boundary-layer. J Atmos Sci 46:1492-1516. doi:10.1175/1520-0469(1989)046<1492:LESOTC >2.0.CO;2

Mason PJ, Brown AR (1999) On subgrid models and filter operations in large eddy simulations. J Atmos Sci 56:2101-2114. doi:10.1175/1520-0469(1999)056<2101:OSMAFO >2.0.CO;2

Mason PJ, Thomson DJ (1992) Stochastic backscatter in large-eddy simulations of boundary-layers. J Fluid Mech 242:51-78. doi:10.1017/S0022112092002271

Meneveau C (1994) Statistics of turbulence subgrid-scale stresses - necessary conditions and experimental tests. Phys Fluids 6:815-833. doi:10.1063/1.868320

Meneveau C, Katz J (2000) Scale-invariance and turbulence models for large-eddy simulation. Annu Rev Fluid Mech 32:1-32. doi:10.1146/annurev.fluid.32.1.1

Mitsuishi A, Hasegawa Y, Kasagi N (2003): Large eddy simulation of mass transfer across an air-water interface at high schmidt numbers. In: The 6th ASME-JSME thermal engineering joint conference, Hawai, USA, paper number TED-AJ03-231.

Moeng CH (1984) A large-eddy-simulation model for the study of planetary boundary-layer turbulence. J Atmos Sci 41:2052-2062. doi:10.1175/1520-0469(1984)041<2052:ALESMF>2.0.CO;2

Moin P, Squires K, Cabot W, Lee S (1991) A dynamic subgrid-scale model for compressible turbulence and scalar transport. Phys Fluids A 3:2746-2757. doi:10.1063/1.858164

O'Sullivan PL, Biringen S, Huser A (2001) A priori evaluation of dynamic subgrid models of turbulence in square duct flow. J Eng Math 40:91-108. doi:10.1023/A:1017552106889

Parlange MB, Eichinger WE, Albertson JD (1995) Regional-scale evaporation and the atmospheric boundary-layer. Rev Geophys 33:99-124. doi:10.1029/94RG03112

Patton EG, Sullivan PP, Moeng CH (2005) The influence of idealized heterogeneity on wet and dry planetary boundary layers coupled to the land surface. J Atmos Sci 62:2078-2097. doi:10.1175/JAS3465.1

Piomelli U (1999) Large-eddy simulation: achievements and challenges. Prog Aerosp Sci 35:335-362. doi:10.1016/S0376-0421(98)00014-1

Pitsch H, Steiner H (2000) Large-eddy simulation of a turbulent piloted methane/air diffusion flame (Sandia flame D). Phys Fluids 12:2541-2554. doi:10.1063/1.1288493

Pope SB (2000) Turbulent flows. Cambridge University Press, Cambridge, UK, 771 pp

Pope SB (2004) Ten questions concerning the large-eddy simulation of turbulent flows. N J Phys 6:771. doi:10.1088/1367-2630/6/1/035

Porte-Agel F (2004) A scale-dependent dynamic model for scalar transport in large-eddy simulations of the atmospheric boundary layer. Boundary-Layer Meteorol 112:81-105. doi:10.1023/B:BOUN. 0000020353.03398 .20

Porte-Agel F, Meneveau C, Parlange MB (1998) Some basic properties of the surrogate subgrid-scale heat flux in the atmospheric boundary layer. Boundary-Layer Meteorol 88:425-444. doi:10.1023/A: 1001521504466

Porte-Agel F, Parlange MB, Meneveau C, Eichinger WE, Pahlow M (2000) Subgrid-scale dissipation in the atmospheric surface layer: Effects of stability and filter dimension. J Hydromet 1:75-87. doi:10.1175/1525-7541(2000)001<0075:SSDITA >2.0.CO;2

Porte-Agel F, Pahlow M, Meneveau C, Parlange MB (2001a) Atmospheric stability effect on subgridscale physics for large-eddy simulation. Adv Water Resour 24:1085-1102. doi:10.1016/ S0309-1708(01)00039-2

Porte-Agel F, Parlange MB, Meneveau C, Eichinger WE (2001b) A priori field study of the subgridscale heat fluxes and dissipation in the atmospheric surface layer. J Atmos Sci 58:2673-2698. doi:10.1175/1520-0469(2001)058<2673:APFSOT>2.0.CO;2

Sagaut P (2003) Large eddy simulation for incompressible flows. Springer-Verlag, Berlin, 426 pp 
Scotti A, Meneveau C, Lilly DK (1993) Generalized Smagorinsky model for anisotropic grids. Phys Fluids A 5:2306-2308. doi:10.1063/1.858537

Shen L, Yue DKP (2001) Large-eddy simulation of free-surface turbulence. J Fluid Mech 440:75-116. doi:10. $1017 /$ S0022112001004669

Smagorinsky J (1963) General circulation experiments with the primitive equations: I. The basic experiment. Mon Weather Rev 91:99-164. doi:10.1175/1520-0493(1963)091<0099:GCEWTP>2.3.CO;2

Stoll R, Porte-Agel F (2006) Dynamic subgrid-scale models for momentum and scalar fluxes in large-eddy simulations of neutrally stratified atmospheric boundary layers over heterogeneous terrain. Water Resour Res 42:W01409. doi:10.1029/2005WR003989

Sullivan PP, McWilliams JC, Moeng CH (2000) Simulation of turbulent flow over idealized water waves. J Fluid Mech 404:47-85. doi:10.1017/S0022112099006965

Sullivan PP, Horst TW, Lenschow DH, Moeng CH, Weil JC (2003) Structure of subfilter-scale fluxes in the atmospheric surface layer with application to large-eddy simulation modelling. J Fluid Mech 482:101-139. doi:10.1017/S0022112003004099

Sullivan PP, Edson JB, Horst TW, Wyngaard JC, Kelly M (2006) Subfilter scale fluxes in the marine surface layer: results from the ocean horizontal array turbulence study (OHATS). In: 17th Symposium on boundary layers and turbulence, San Diego, U.S.A.

Sullivan PP, Mcwilliams JC, Melville WK (2007) Surface gravity wave effects in the oceanic boundary layer: large-eddy simulation with vortex force and stochastic breakers. J Fluid Mech 593:405-452. doi:10. $1017 /$ S002211200700897X

Tao B, Katz J, Meneveau C (2002) Statistical geometry of subgrid-scale stresses determined from holographic particle image velocimetry measurements. J Fluid Mech 457:35-78. doi:10.1017/S0022112001007443

Tong CN, Wyngaard JC, Brasseur JG (1999) Experimental study of the subgrid-scale stresses in the atmospheric surface layer. J Atmos Sci 56:2277-2292. doi:10.1175/1520-0469(1999)056<2277:ESOTSS>2.0.CO;2

Webb EK, Pearman GI, Leuning R (1980) Correction of flux measurements for density effects due to heat and water-vapor transfer. Quart J Roy Meteorol Soc 106:85-100. doi:10.1002/qj.49710644707

Wood N (2000) Wind flow over complex terrain: a historical perspective and the prospect for large-eddy modelling. Boundary-Layer Meteorol 96:11-32. doi:10.1023/A:1002017732694

Yimer I, Campbell I, Jiang LY (2002) Estimation of the turbulent Schmidt number from experimental profiles of axial velocity and concentration for high-Reynolds-number jet flows. Can Aeronaut Space J 48: 195-200 